\title{
Alte Deiche
}

Liebe Leserinnen, liebe Leser,

bekanntlich schützt man sich gegen Lärm auf zweierlei Weise: Man verstopft sich die Ohren, oder man stellt den Lärm ab. Man macht sein Haus schalldicht oder dämpft den Straßenlärm. Lärmschutzwände werden entweder direkt an der lärmenden Straße errichtet, oder man legt sie unmittelbar vor seinem Grundstück an. Anders angelegt sind Lärmschutzwände praktisch nutzlos. Gleichermaßen schützt man sich gegen Hochwasser, entweder durch Deiche am Gewässerrand oder durch Sandsäcke vor den eigenen Kellerfenstern. Man begegnet einer Bedrohung entweder offensiv an der Quelle oder defensiv dort, wo sie Schaden anzurichten droht. In der Regel tut man beides, nicht aber irgendein zwischen Quelle und Ziel Gelegenes. Beide Arten von Maßnahmen gelten zwar der selben Bedrohung; aber sie unterscheiden sich voneinander prinzipiell. Aktive Maßnahmen dienen dem Interesse aller; sie werden deshalb von der Gemeinschaft getroffen. Mit passiven Maßnahmen schützt sich der Einzelne selbst. Oft braucht er sich nicht zu bemühen, weil die aktive Lärmdämpfung hinreicht. Anderenfalls mag er zusätzlich sein Haus dicht machen. Damit sind die Möglichkeiten ausgeschöpft. Wenn die Bedrohung dennoch nicht weicht, muss er sich mit ihr abfinden oder die Flucht ergreifen.

Von Naturereignissen abgesehen, ist aber die Gemeinschaft als solche das Bedrohliche. Kann sie mich aber gleichzeitig bedrohen und gegen die Bedrohung schützen? Es kommt darauf an: Bei der Bedrohungen durch Böses, etwa durch Kriminalität, sind die Verhältnisse klar: Das Böse wird wie Lärm und Hochwasser bekämpft, passiv durch private defensive Vorkehrungen und aktiv durch den Staat. Das ist auch für den Datenschutz im privaten Bereich sinnvoll. Der Staat baut Dämme gegen die überbordende Verarbeitung personenbezogener Daten, um Freiheitsrechte zu schützen und gibt jedermann zur persönlichen Verteidigung Rechte und Mittel an die Hand. Sie sind die Sandsäcke, mit denen jeder seinen persönlichen Bereich abschirmen kann.

Doch hat der Staat nicht nur Schutzaufgaben. Er regiert und übt dabei gegen jedermann Macht aus. Der Logik der Machtausübung folgend, misstraut er den Regierten, macht sie sich transparent und behindert sie diesbezüglich am Selbstschutz. Er tut dies aus guten Gründen zur Gewährleistung von Gerechtigkeit und kollektiver Sicherheit. Gleichwohl fühlt sich der Mensch dabei - ob zu Recht oder zu Unrecht - bedroht, etwa von der Polizei, dem Finanzamt oder dem Gerichtsvollzieher; darüber hinaus auch von Atomkraftwerken, öffentlichen Baumaßnahmen etc. Im Geschäft mit dem Staat gibt es also Bedrohungen, bei denen der dagegen gerichtete aktive Schutz sich gegen den staatlichen Machtanspruch richtet. Der Staat muss den Staat zwingen, bei seiner Machtausübung die Freiheitsrechte der Regierten einzuhalten. Ein Dilemma der Demokratie. Das führt zum Konflikt staatlicher Machtausübung mit den Freiheitsrechten. Es betrifft jederlei Bedrohung durch den Staat und trifft besonders auf dessen Datenverarbeitung zu.

Als in den 60er-Jahren die Demokratiebewegung auf den Datenschutz kam, konzentrierte sie sich zunächst auf den Staat. Der Datenschutz orientierte sich an der Datenverarbeitung. Diese hatte sich aber auch im privaten Bereich eingeführt. So gerieten die beiden Bereiche - unter der Gefahr der Vermengung - trotz ihrer prinzipiellen Verschiedenheit in einen Topf. Seit den 70er-Jahren ist aber die Technik dedizierter geworden; die schlichte Datenverarbeitung eignet sich immer weniger als gemeinsamer Nenner. Neue bereichsspezifische Bedrohungen sprengen die alten Konzepte. Eine Flut nach der anderen. Kein Verlass auf die alten Deiche. Ich meine, liebe Leserinnen und Leser, der Datenschutz - aktiv wie passiv - will neue dedizierte Konzepte.

Mit freundlichen Grüßen, Ihr

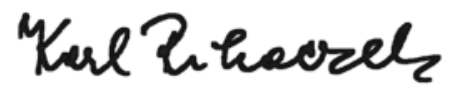

